\title{
EXTRATO DE HIBISCO-COLIBRI COMO INIBIDOR VERDE DE CORROSÃO DO AÇO-CARBONO EM ÁCIDO SULFÚRICO
}

\author{
Arthur Valbon , Bruno F. Ribeiro ${ }^{\mathrm{a}}$, Maria Augusta F. Soares ${ }^{\mathrm{a}}$, Márcia C. C. de Oliveira ${ }^{\mathrm{a}}$, Marcelo A. Neves ${ }^{\mathrm{b}}$ e Aurea \\ Echevarria*,a, (i) \\ aDepartamento de Química Orgânica, Universidade Federal Rural do Rio de Janeiro, 23890-000 Seropédica - RJ, Brasil \\ 'Departamento de Física, Universidade Federal Rural do Rio de Janeiro, 23890-000 Seropédica - RJ, Brasil
}

Recebido em 05/03/2019; aceito em 03/06/2019; publicado na web em 26/06/2019

\begin{abstract}
HIBISCO-COLIBRI EXTRACT AS GREEN CORROSION INHIBITOR FOR CARBON STEEL IN SULFURIC ACID. For this work, a hydroalcoholic extract was prepared from the aerial parts of the Malvaviscus arboreus (Malvaceae) species, popularly known as hibisco-colibri in Brazil; and then, investigated by infrared and NMR spectroscopy. A class of special metabolites containing a porphyrin skeleton and pyrrole units was determined by typical absorption spectra, mainly ${ }^{1} \mathrm{H}$ and ${ }^{13} \mathrm{C}$ NMR, thus indicating the capability of showing anti-corrosion effect. Therefore, the $M$. arboreus extract was examined as a corrosion inhibitor for AISI 1020 carbon steel in $\mathrm{H}_{2} \mathrm{SO}_{4} 1 \mathrm{~mol} \mathrm{~L}^{-1}$, using a mass loss (gravimetric) technique. Results showed good corrosion inhibition efficiency, at both time and temperature variation. The maximum efficiency observed at $500 \mathrm{ppm}$ was $97.5 \%$, at $3 \mathrm{~h}, 24 \mathrm{~h}$ and $48 \mathrm{~h}$. The surface morphology was analyzed by scanning electron microscopy (SEM), through which a higher homogeneity was observed in the presence of the M. arboreus extract. The hydroalcoholic extract of hibisco-colibri can be considered an efficient green agent for preventing corrosion.
\end{abstract}

Keywords: Malvaviscus arboreus; corrosion inhibitor; ecofriendly inhibitor.

\section{INTRODUÇÃO}

Processos corrosivos são espontâneos e acontecem pela ação química ou eletroquímica do meio ambiente promovendo, geralmente, a degradação de materiais metálicos ou não, que podem ser intensificados por esforços mecânicos. Corrosão é um fenômeno prejudicial para as indústrias, edifícios, pontes rodoviárias e ferroviárias, residências entre outros. Na indústria a corrosão atua afetando as propriedades físicas e químicas do metal ou suas ligas, tornando-os inapropriados para a manutenção adequada das atividades industriais, devido ao risco de acidentes, contaminação de produtos, desastres ambientais, etc. ${ }^{1}$ Estruturas metálicas de aplicação industrial são frequentemente expostas a meios que facilitam os processos corrosivos, como, por exemplo, a água do mar e o solo, sendo danificadas antes do tempo previsto, o que gera prejuízos significativos. ${ }^{2}$

Devido aos inúmeros prejuízos causados pela corrosão, o desenvolvimento de métodos cada vez mais eficazes de proteção torna-se necessário. $\mathrm{O}$ uso de agentes inibidores tem sido uma alternativa bastante promissora e, os inibidores orgânicos mostram importante eficiência. Os agentes inibidores são substâncias que, quando adicionadas ao meio corrosivo, evitam ou diminuem o desenvolvimento das reações de corrosão. Estes compostos orgânicos adsorvem sobre a superfície dos metais e ligas através de seus heteroátomos (geralmente oxigênio, nitrogênio e enxofre) e $\pi$-elétrons e, formam uma barreira de superfície que protege os metais da degradação corrosiva. ${ }^{3,4}$

A pesquisa no campo de inibidores orgânicos de corrosão tem levado em consideração a redução de custos de produção, o aumento da eficiência e a utilização de inibidores ecologicamente corretos (eco-friendly). A investigação visando a utilização de extratos vegetais ou produtos naturais isolados na inibição da corrosão surgiu, no início deste século, como uma alternativa para minimizar o impacto ambiental causado pelas indústrias. ${ }^{5-7}$

*e-mail: echevarr@ufrrj.br
Diversas espécies vegetais são ricas em substâncias contendo anéis aromáticos, insaturações, átomos de oxigênio, nitrogênio e enxofre, capazes de interagir e até se complexar com metais, possibilitando a redução dos processos corrosivos. As principais classes presentes nos extratos incluem os ácidos orgânicos, as quinonas, os flavonoides, as catequinas, terpenóides, alcaloides, além de aminoácidos, proteínas, polissacarídeos e vitaminas que não têm efeitos adversos significativos sobre os organismos vivos e o meio ambiente. ${ }^{8,9}$

Assim, devido ao baixo custo, natureza renovável, biodegradabilidade e facilidade de aplicação, vários extratos vegetais têm sido investigados quanto aos efeitos anticorrosivos. $\mathrm{O}$ extrato da borra de café descrito por Torres et al, 2011, apresentou eficiência máxima de $97 \%$ de inibição da corrosão, utilizando 400 ppm de extrato em meio de $\mathrm{HCl} 1 \mathrm{~mol} \mathrm{~L}^{-1}$ frente ao aço-carbono. ${ }^{10}$ Ostovari et al, 2009, obteve $92 \%$ de eficiência de inibição da corrosão utilizando extrato de hena (Lawsonia inermes) na concentração de 1200 ppm, em aço-carbono e meio de ácido clorídrico. ${ }^{11}$

O extrato das folhas de quebra-pedra (Phyllanthus amarus) apresentou $76 \%$ de eficiência de inibição da corrosão na concentração de $20 \%$ em meio de hidróxido de sódio. ${ }^{12}$ A pimenta-longa (Piper longum) também foi estudada como potencial inibidor da corrosão de alumínio e a eficiência de inibição foi de $94 \%$ na concentração de 400 ppm, também em presencça de hidróxido de sódio. ${ }^{13}$

Os extratos das folhas de jasmim amarelo ${ }^{14}$ (Jasminum nudiflorum) e amora-da-índia ${ }^{15}$ (Morinda tinctoria) apresentaram bons resultados na inibição da corrosão em alumínio em meio de ácido clorídrico, apresentando $93 \%$ na concentração de 1000 ppm ${ }^{14}$ e $97 \%$ na concentração de $7 \%$ (v/v), ${ }^{15}$ respectivamente.

A espécie Malvaviscus arbororeus Cav., chamada de hibiscocolibri, pertence à família Malvaceae, é nativa do México e norte da América do Sul, mas amplamente distribuída em toda América tropical, sendo utilizada, principalmente, como planta ornamental em cercas-vivas ou isolada devido ao seu manejo simples e floração durante todo o ano. Embora não existam registros na literatura de estudos dessa espécie quanto aos efeitos frente à corrosão, recentemente, 
outras espécies da família Malvaceae e do gênero Hibiscus, também vulgarmente conhecidas como hibiscos, tiveram seus extratos avaliados quanto à atividade anticorrosiva.

O extrato aquoso das folhas de Hibiscus cannabinus ${ }^{16}$ foi avaliado em aço-carbono em meio de ácido sulfúrico, apresentando uma eficiência de inibição da corrosão de $93 \%$ utilizando a concentração de 3000 ppm. A espécie $H$. sabdariffa foi investigada através de seu extrato aquoso das folhas frente ao aço-carbono em ácido fosfórico, ${ }^{17}$ e em liga de aço-leve em ácido clorídrico, ${ }^{18}$ mostrando eficiência anticorrosiva da ordem de $90 \%$, e em alumínio em meio de ácido sulfúrico ${ }^{19}$ com eficiência máxima de $88 \%$.

Diante desse contexto, o objetivo deste trabalho foi avaliar a eficiência anticorrosiva do extrato hidro-alcoólico de Malvaviscus arboreus frente ao aço-carbono AISI 1020 em ácido sulfúrico utilizando o método gravimétrico em diferentes temperaturas, visando o cálculo dos parâmetros termodinâmicos (constante de adsorção, energia de ativação, entalpia e entropia), e a microscopia eletrônica de varredura (SEM - Scanning Electron Microscopy). Além disso, realizar a prospecção das principais classes de metabólitos especiais presentes no extrato através dos espectros de infravermelho e RMN de ${ }^{1} \mathrm{H}$ e ${ }^{13} \mathrm{C}$.

\section{PARTE EXPERIMENTAL}

\section{Coleta e identificação do material vegetal}

As folhas de Malvaviscus arboreus Cav. foram coletadas em outubro de 2016 no bairro Ecologia, localizado no Campus de Seropédica da Universidade Federal Rural do Rio de Janeiro (UFRRJ), RJ. Uma amostra foi comparada com a exsicata $n^{\circ}$ RBR 44958 depositada no Herbário do Departamento de Botânica da UFRRJ, identificada por Thiago de Azevedo Amorim.

\section{Preparação do extrato vegetal}

O extrato foi preparado a partir de $627,6 \mathrm{~g}$ das folhas, in natura, de $M$. arboreus por maceração em etanol:água (9:1, v:v) durante 15 dias com troca semanal do solvente. Após esse período o extrato foi filtrado e concentrado em rotaevaporador, obtendo-se o extrato cru seco $(24,85 \mathrm{~g})$ com $4 \%$ de rendimento.

\section{Prospecção por infravermelho, RMN de ${ }^{1} \mathrm{H}$ e ${ }^{13} \mathrm{C}$}

O espectro de infravermelho, utilizando-se a técnica de ATR, foi obtido em espectrofotômetro da Bruker modelo Vertex 70. Os espectros de RMN de ${ }^{1} \mathrm{H}$ e ${ }^{13} \mathrm{C}$ do extrato seco de $M$. arboreus foram obtidos em $\mathrm{CDCl}_{3}$ em espetrômetro Bruker operando em 500 e $125 \mathrm{MHz}$, respectivamente.

\section{Avaliação anticorrosiva}

O potencial anticorrosivo do extrato de $M$. arboreus foi testado frente ao aço carbono AISI 1020, realizado em solução de $\mathrm{H}_{2} \mathrm{SO}_{4}$ $1 \mathrm{~mol} \mathrm{~L}^{-1}$, na ausência (branco) e na presença de cinco concentrações do extrato, utilizando a técnica gravimétrica de perda de massa.

Os corpos de prova de dimensão de $3 \mathrm{~cm} \times 3 \mathrm{~cm} \times 0,15 \mathrm{~cm}$, foram polidos com lixas d'agua de diferentes granulometrias (150, 400, 800 mesh), em seguida as peças foram desengorduradas com etanol, secas com soprador de ar e finalmente pesadas.

Os ensaios gravimetricos foram realizados em diferentes temperaturas $(308,318,328,338 \mathrm{~K})$ na concentração de 500 ppm, com 3 horas de imersão. A eficiencia de inibição da corrosão também foi observada variando o tempo de imersão (3, 24 e 48 horas), nas concentraçoes de $500,125,50,35,15$ ppm. $^{10}$
Cada medida foi realizada em triplicata. Após o tempo de imersão, os corpos de prova foram lavados com agua destilada, submetidos a banho de ultrassom imersos em etanol, secos e, finalmente, pesados. ${ }^{10,13,15,19}$

A eficiência de inibição da corrosão $E I_{\mathrm{PM}}$ foi calculada de acordo com a Equação 1:

$$
E I_{P M}=\frac{W_{0}-W}{W_{0}} \times 100
$$

em que $W_{0}$ e $W$ são as taxas de corrosão na ausência e presença de inibidor, respectivamente.

\section{Análise de microscopia eletrônica de varredura}

As amostras de aço carbono de $3,0 \times 3,0 \times 0,1 \mathrm{~cm}$ foram lixadas e lavadas com etanol, secas e imersas em presença e ausência de 500 ppm de extrato frente $\mathrm{H}_{2} \mathrm{SO}_{4} 1 \mathrm{~mol} \mathrm{~L}^{-1}$, durante $24 \mathrm{~h}$ em temperatura ambiente. Os corpos de prova foram removidos, lavados com água, etanol e secos. As medições foram feitas usando uma Hitachi Microscópio de mesa TM 3000..$^{20-22}$

\section{RESULTADOS E DISCUSSÃO}

\section{Prospecção por infravermelho, $\mathrm{RMN}$ de ${ }^{1} \mathrm{H}$ e ${ }^{13} \mathrm{C}$}

O extrato hidroalcoólico das folhas de $M$. arboreus foi submetido à prospecção das principais classes de metabólitos especiais utilizando métodos espectroscópicos de rotina. A espectroscopia é fundamental na elucidação estrutural de substâncias naturais e contribui com a ampliação de um banco de dados, o qual facilita a identificação dessas substâncias, mesmo quando estas fazem parte de uma mistura.

$\mathrm{O}$ espectro de infravermelho (Figura 1) informou bandas de absorção na região 3315, 3010, 2925 e $2854 \mathrm{~cm}^{-1}$, as quais são associadas ás vibrações de deformação axial que ocorrem nas ligações entre $\mathrm{N}-\mathrm{H}, \mathrm{C}-\mathrm{H}$ (olefinico), $\mathrm{CH}_{2}$ e $\mathrm{CH}_{3}$, respectivamente. As bandas associadas às vibrações de deformação axial de ligações duplas estão representadas no espectro em 1737 ( $\mathrm{C}=\mathrm{O}$ da função éster), 1629 e $1583 \mathrm{~cm}^{-1}(\mathrm{C}=\mathrm{C})$. Bandas de confirmação de vibrações de ligação $\mathrm{C}-\mathrm{O}$ de éster e $\mathrm{RCH}=\mathrm{CH}_{2}$ aparecem em $1259 \mathrm{~cm}^{-1}$ e $931-900 \mathrm{~cm}^{-1}$, respectivamente.

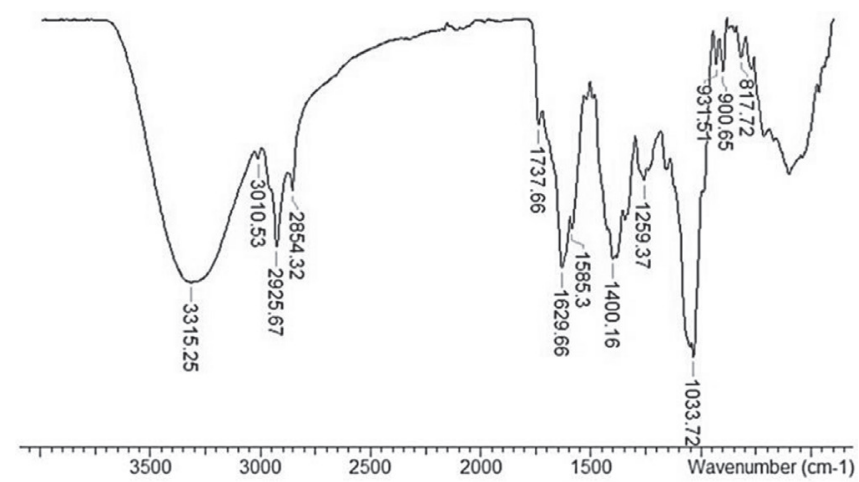

Figura 1. Espectro de infravermelho obtido do extrato hidroalcoólico das folhas de M. arboreus

A espectroscopia de ressonância magnética nuclear $(\mathrm{RMN})$ pode ser usada como ferramenta de rotina na análise do perfil de metabólitos presentes em extratos obtidos de plantas, pois cada núcleo de hidrogênio e carbono, quimicamente distinto em cada metabolito presente em uma amostra, exibirá um sinal de RMN com uma frequência de ressonância característica. Nesse sentido, essa técnica foi usada 
para determinar a composição química do extrato hidroalcoólico das folhas de $M$. arboreus.

$\mathrm{O}$ espectro RMN de ${ }^{1} \mathrm{H}$ (Figura 2) e de ${ }^{13} \mathrm{C}$-DEPTQ (Figura 3) apresentaram sinais característicos de esqueleto cíclico tertrapirrólico, presente no sistema heterocíclico das porfirinas..$^{23,24}$ Os singletos em $\delta_{\mathrm{H}} 8.58,9.42$ e 9.56 ppm correspondem aos hidrogênios H-20, $\mathrm{H}-5$ e H-10, os quais estão ligados a carbonos $\mathrm{sp}^{2}$ com sinais em $\delta_{\mathrm{C}} 93.15(\mathrm{C}-20), 97.57$ (C-5) e 104.48 (C-10) ppm, presentes no sistema aromático porfirínico. Os sinais do grupo vinílico ligado a $\mathrm{C}-3\left(\delta_{\mathrm{C}} 137.96\right)$ estão representados em $\delta_{\mathrm{CH}} 8.02 / 123.04\left(\mathrm{H}-3^{1} / \mathrm{C}-3^{1}\right)$ e $6.21,6.24 / 122.85\left(2 \mathrm{H}-3^{2} / \mathrm{C}-3^{2}\right)$.

Os sinais de hidrogênios e carbonos referentes a duas metilas presentes nas porfirinas estão representados como singletos em $\delta_{\mathrm{CH} 3} 3.27 / 11.29\left(\mathrm{H}-2^{1} / \mathrm{C}-2^{1}\right) ; 3.43 / 12.13\left(\mathrm{H}-12^{1} / \mathrm{C}-12^{1}\right)$. Os hidrogênios metílicos que acoplam com os grupos metilênico $\left(\delta_{\mathrm{CH} 2} 3.71 / 19.49\right.$, H-8 $\left.1 / \mathrm{C}-8^{1}\right)$ e metínico $\left(\delta_{\mathrm{CH} 2} 3.90 / 50.91, \mathrm{H}-18 / \mathrm{C}-18\right)$ estão representados em $\delta_{\mathrm{CH} 3} 1.62(\mathrm{t}) / 17.45\left(\mathrm{H}-8^{2}\right.$ e C- $\left.8^{2}\right)$ e $1.72(\mathrm{~d}) / 22.63\left(\mathrm{H}-18^{1}\right.$ e
C-18 ${ }^{1}$ ). Os sinais em $\delta_{\mathrm{H}} 4.14$ ppm para $\mathrm{H}-17, \delta_{\mathrm{C}} 50.90$ ppm para C-17 e o grupo fitolato representado pelos sinais em $\delta_{\mathrm{C}} 29.69 \mathrm{ppm}\left(\mathrm{C}-17^{1}\right)$, $\delta_{\mathrm{C}} 31.93 \mathrm{ppm}\left(\mathrm{C}-17^{2}\right), \delta_{\mathrm{C}} 173.95 \mathrm{ppm}\left(\mathrm{C}-17^{3}\right), \delta_{\mathrm{C}} 17.45,19.72$ e 27.90 ppm (metilas do grupo fitil) e $\delta_{\mathrm{H}} 0.88-1.69,5.32$ (d) e 5.38 (t) ppm corroboram com os valores compatíveis presentes no núcleo porfirínico.

A prospecção das classes de metabólitos por infravermelho e RMN de ${ }^{1} \mathrm{H} \mathrm{e}{ }^{13} \mathrm{C}$ possibilitou identificar a principal classe presente no extrato hidroalcoolico de $M$. arboreus, como sendo a das porfirinas formadas com anéis tetra-pirrolicos também presentes em extratos de outras espécies vegetais e, que apresentaram efeito quelante frente aos íons de ferro, ${ }^{25,26}$ indicando assim a possibilidade de apresentar efeito anticorrosivo.

\section{Atividade anticorrosiva}

A eficiência de inibição da corrosão $\left(\eta_{\mathrm{PM}}\right)$ do extrato hidro alcoolico de M. arboreus frente ao aço-carbono AISI 1020 em ácido

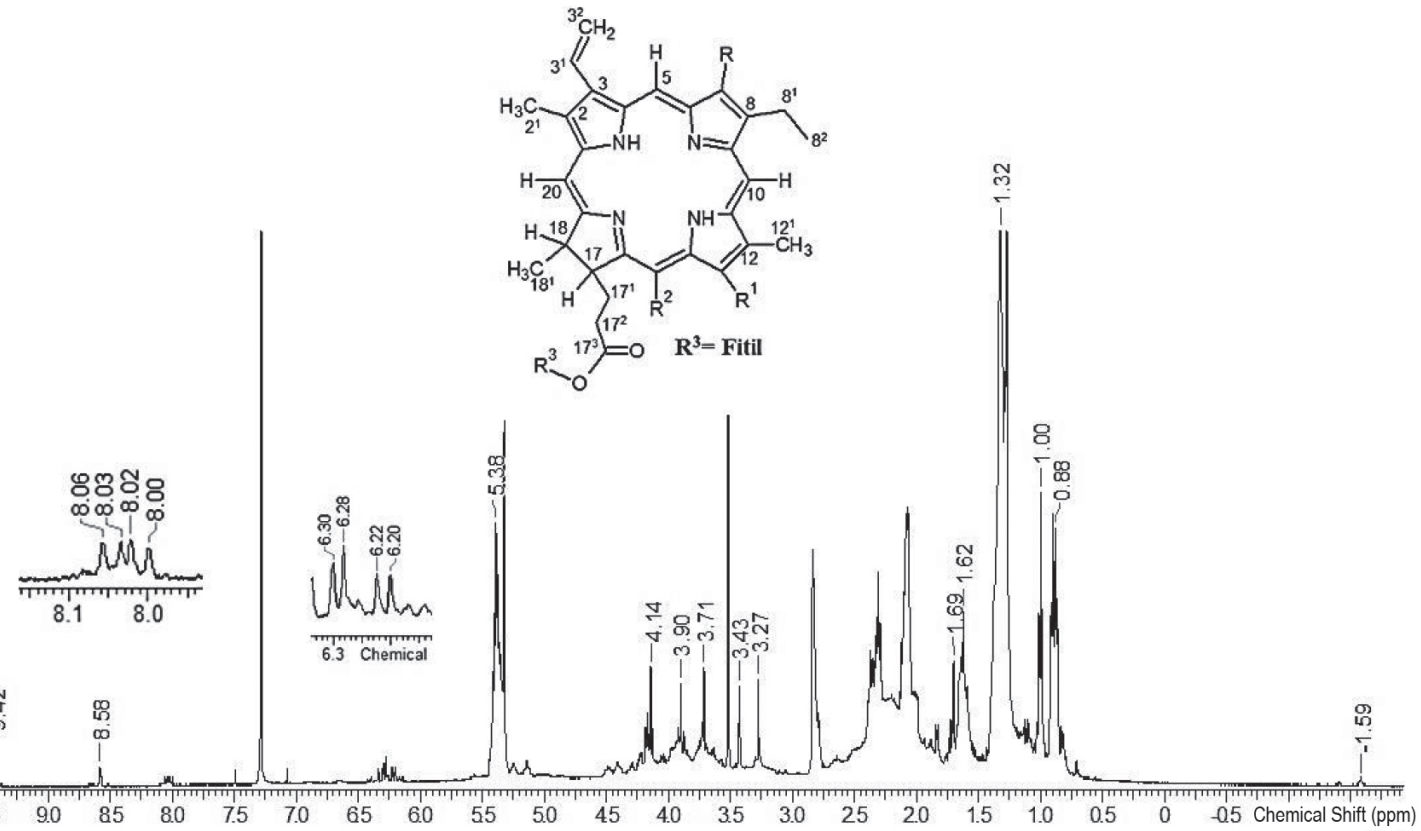

Figura 2. Espectro de $\mathrm{RMN}^{1} \mathrm{H}\left(500 \mathrm{MHz}^{\mathrm{C}} \mathrm{CDCl}_{3}\right)$ do extrato hidroalcoólico das folhas de M. arboreus

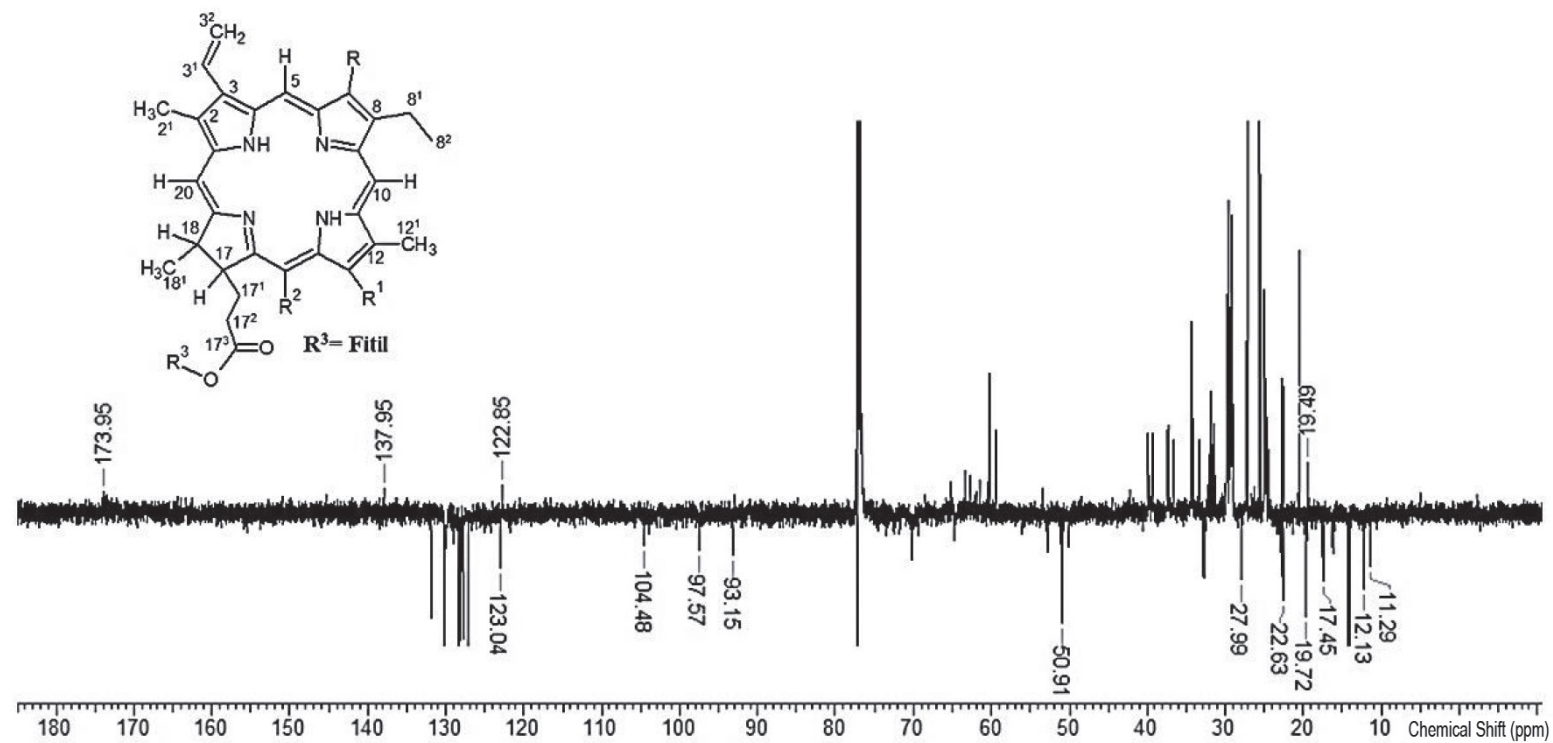

Figura 3. Espectro de $R M N C^{13}-D E P T Q\left(125 \mathrm{MHz}, \mathrm{CDCl}_{3}\right)$ do extrato hidroalcoólico das folhas de M. arboreus 
Tabela 1. Perda de massa em $\mathrm{H}_{2} \mathrm{SO}_{4} 1$ mol L-1 em ausência e presença de extrato hidro alcoólico de $M$. arboreus nos tempos de 3, 24 e 48 horas nas concentrações de 500, 125, 50 e 35 ppm

\begin{tabular}{|c|c|c|c|c|c|c|}
\hline \multirow{2}{*}{$\begin{array}{l}\text { Concentração } \\
(\mathrm{ppm})\end{array}$} & \multicolumn{2}{|c|}{3 horas } & \multicolumn{2}{|c|}{24 horas } & \multicolumn{2}{|c|}{48 horas } \\
\hline & $\begin{array}{c}W_{\text {corr }} \\
\left(\mathrm{mg} \mathrm{cm}^{-2} \mathrm{~h}^{-1}\right)\end{array}$ & $E I(\%)$ & $\begin{array}{c}W_{\text {corr }} \\
\left(\mathrm{mg} \mathrm{cm}^{-2} \mathrm{~h}^{-1}\right)\end{array}$ & $E I(\%)$ & $\begin{array}{c}W_{\text {corr }} \\
\left(\mathrm{mg} \mathrm{cm}^{-2} \mathrm{~h}^{-1}\right)\end{array}$ & $E I(\%)$ \\
\hline Branco & 6,364 & - & 2,89 & - & 2,622 & - \\
\hline 35 & 0,806 & 87,5 & 1,865 & 35,7 & 1,938 & 26,1 \\
\hline 50 & 0,315 & 95,0 & 0,461 & 84,1 & 1,826 & 30,3 \\
\hline 125 & 0,249 & 96,0 & 0,138 & 95,2 & 0,131 & 95,0 \\
\hline 500 & 0,158 & 97,5 & 0,070 & 97,5 & 0,063 & 97,5 \\
\hline
\end{tabular}

sufúrco $1 \mathrm{~mol} \mathrm{~L}^{-1}$ foi avaliada pela técnica gravimétrica de perda de massa, inicialmente realizada na temperatura de $308 \mathrm{~K}$, nas concentrações de 500, 125, 50, 35, 15 ppm, com tempo de imersão de 3, 24 e 48 horas.

Posteriormente, a eficiência de inibição da corrosão foi verificada variando a temperatura do meio, em 308, 318, 328, $338 \mathrm{~K}$, com tempo de imersão de 3 horas e concentração de 500 ppm.

\section{Tempo de imersão}

A variação do tempo de imersão é uma ferramenta importante para análise da estabilidade do inibidor e, consequentemente, do filme formado na superfície do metal. ${ }^{27}$

A Tabela 1 mostra os resultados obtidos nos experimentos de perda de massa nos tempos de 3, 24 e 48 horas, nas concentrações de $500,125,50,35$ e 15 ppm.

É possível observar um aumento da eficiência de inibição da corrosão com o aumento da concentração de extrato. Com o aumento do tempo de imersão, nas concentrações de 50 e 35 ppm a eficiência de inibição decai com o tempo. Porém, a eficiência se mantém estável nos tempos de 3, 24 e 48 horas, nas concentrações de 500 e 125 ppm. Tal comportamento deve ser atribuído à estabilidade do filme adsorvido na superfície metálica, possivelmente devido à interação entre os pares de elétrons livres dos nitrogênios e ligações duplas dos anéis pirrólicos (presentes nos compostos identificados na prospecção espectroscópica) com a superfície metálica. ${ }^{28-30}$

As interações entre o extrato de $M$. arboreus e a superfície metálica podem ser estudadas pela isoterma de adsorção correspondente, que é determinada pela relação entre concentração de inibidor e grau de cobertura da superfície.

O grau de cobertura $(\theta)$ é diretamente proporcional à eficiência de inibição da corrosão e pode ser calculado de acordo com a Equação 2. 10,21,22

$$
\theta=\frac{W_{0}-W}{W_{0}}
$$

em que $W_{0}$ e $W$ são as taxas de corrosão na ausência e presença de inibidor, respectivamente.

No presente trabalho, a isoterma que melhor se adequou ao sistema de adsorção inibidor/superfície foi a de Langmuir. Esse ajuste foi verificado pelo coeficiente de correlação linear (r), obtido pela regressão linear do gráfico de $\mathrm{C} / \theta$ versus $\mathrm{C}$. A isoterma de Langmuir é representada pela Equação 3: ${ }^{10,21,28}$

$$
\frac{C}{\theta}=\frac{1}{K_{a d s}}+C
$$

em que $C$ é a concentração do inibidor, $K_{\text {ads }}$ é a constante de equilíbrio e $\theta$ é o grau de cobertura da superfície (obtido no tempo de $3 \mathrm{~h}$, nas concentrações de 500, 125, 50 e 35 ppm).
Na Figura 4 é possível observar o gráfico obtido pela isoterma de Langmuir.

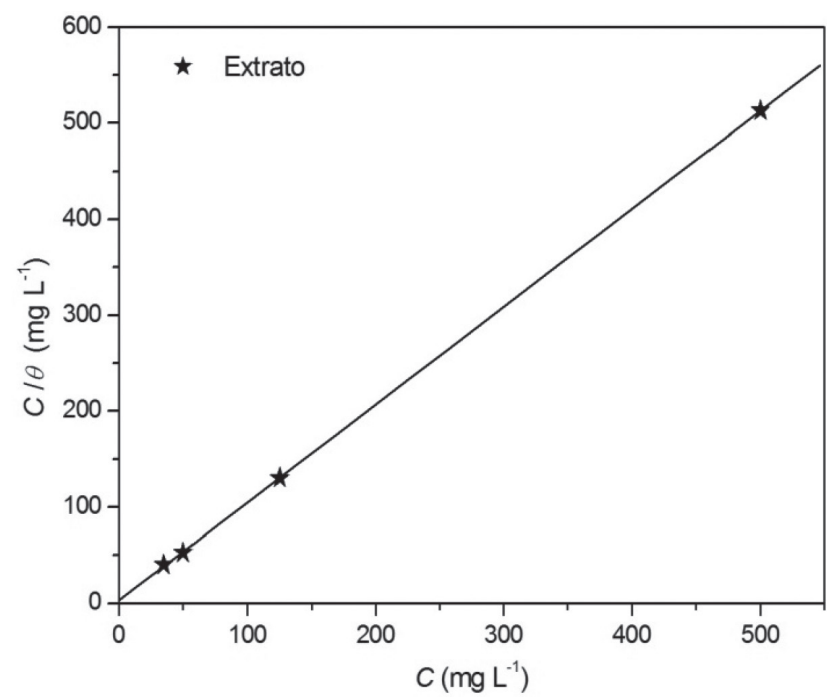

Figura 4. Isoterma de Langmuir para o extrato de M. arboreus em aço carbono AISI 1020 em solução de $\mathrm{H}_{2} \mathrm{SO}_{4} 1 \mathrm{~mol} \mathrm{~L}^{-1}$

Após a linearização, foram obtidos bons valores de coeficiente de correlação e slope, que são 0,9999 e 1,01, respectivamente. O valor de slope próximo da unidade indica que a adsorção do extrato obedece à isoterma de Langmuir. ${ }^{14}$ Tal isoterma assume que a adsorção ocorre em sítios específicos e homogêneos na superfície do adsorvente. ${ }^{30}$

$\mathrm{O}$ valor de constante de adsorção $K_{\text {ads }}$ foi de $0,330 \mathrm{~L} \mathrm{mg}^{-1}$. Na literatura, os valores de $K_{\text {ads }}$ obtidos para extratos vegetais são comparáveis com o obtido neste trabalho, sendo para bons inibidores de corrosão em meio ácido, por exemplo, 0,1127 $\mathrm{L} \mathrm{mg}^{-1}$ para o extrato das sementes de mamão papaia, e $0,0580 \mathrm{~L} \mathrm{mg}^{-1}$ para o extrato da borra de café torrado, entre outros. ${ }^{10,31} \mathrm{O}$ valor de $\Delta G_{\text {ads }}$ é um parâmetro importante para a avaliação da interação entre o agente inibidor e a superfície do metal. No entanto, trata-se de um parâmetro que envolve a massa molecular e, no caso de extratos vegetais, essas moléculas não são totalmente conhecidas, não sendo possível, portanto, realizar sua determinação. ${ }^{10,30-37}$

\section{Efeito da temperatura}

A temperatura de trabalho pode influenciar a eficiência da inibição de corrosão e, geralmente, a taxa de corrosão aumenta com um aumento da temperatura. Esse fato se dá devido à evolução acelerada de $\mathrm{H}_{2}$ que, consequentemente, aumenta a taxa de dissolução do metal.

Dessa forma, no presente trabalho, foi estudado o comportamento da eficiência de inibição da corrosão do extrato de $M$. arboreus, na concentração de 500 ppm para as temperaturas de 308, 318, 328 e $338 \mathrm{~K}$, durante 3 horas de imersão. 
Na Tabela 2 é possível observar os resultados obtidos nos experimentos com a variação de temperatura.

Tabela 2. Perda de massa em $\mathrm{H}_{2} \mathrm{SO}_{4} 1 \mathrm{~mol} \mathrm{~L}^{-1}$ em ausência e presença de extrato hidro alcoólico de $M$. arboreus nas temperaturas de 308, 318, 328 e $338 \mathrm{~K}$ na concentração de 500 ppm por 3 horas

\begin{tabular}{lccc}
\hline \multirow{2}{*}{ Temperatura (K) } & Branco & \multicolumn{2}{c}{ Extrato } \\
\cline { 2 - 4 } & $\begin{array}{c}W_{\text {corr }} \\
\left(\mathrm{mg} \mathrm{cm}^{-2} \mathrm{~h}^{-1}\right)\end{array}$ & $\begin{array}{c}W_{\text {corr }} \\
\left(\mathrm{mg} \mathrm{cm}^{-2} \mathrm{~h}^{-1}\right)\end{array}$ & $E I(\%)$ \\
\hline 308 & 6,36 & 0,158 & 97,5 \\
318 & 10,74 & 0,382 & 96,5 \\
328 & 17,20 & 0,717 & 96,0 \\
338 & 23,23 & 1,540 & 93,5 \\
\hline
\end{tabular}

Observa-se o aumento da taxa de corrosão na ausência do inibidor (Branco). Porém, quando o ensaio é realizado na presença de 500 ppm de extrato de $M$. arboreus, a eficiência de inibição se mantém praticamente constante, com exceção de $338 \mathrm{~K}$, em que é observada uma eficiência de 93,5\%. Apesar desse comportamento, é possível afirmar que o filme adsorvido é estável até $328 \mathrm{~K}$, e que sua função inibidora não foi comprometida.

A partir dos resultados mostrados na Tabela 2 foi construído o gráfico de Arrhenius, ln $W_{\text {corr }}$ versus $1 / \mathrm{T}$, que pode ser observado na Figura 5.

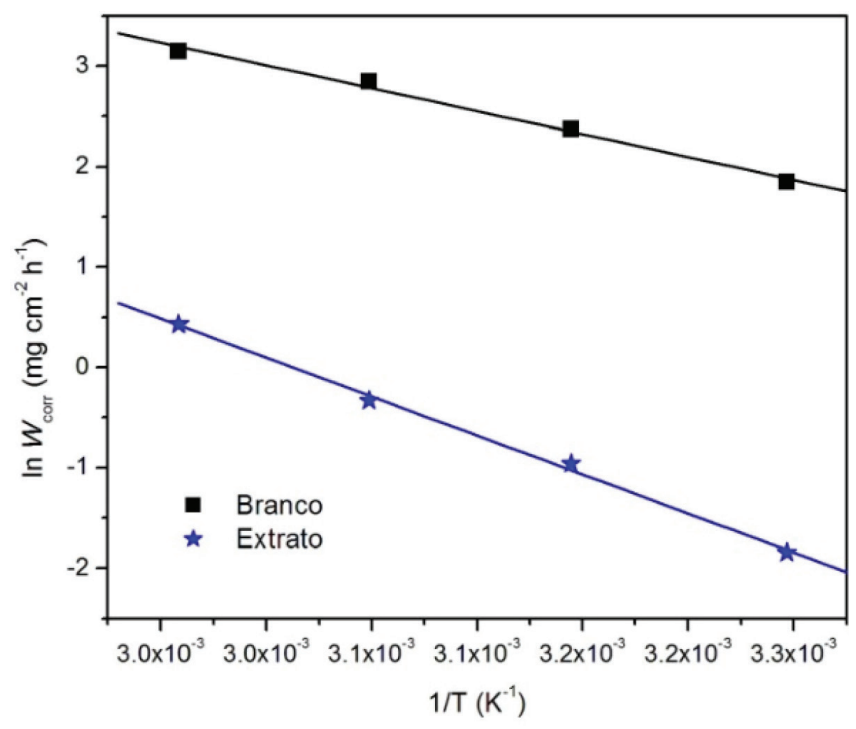

Figura 5. Gráfico de Arrhenius em função da taxa de corrosão e temperatura para o aço carbono AISI 1020 em solução de $\mathrm{H}_{2} \mathrm{SO}_{4} 1 \mathrm{~mol} \mathrm{~L}^{-1}$ na ausência (Branco) e na presença de 500 ppm de M. arboreus (Extrato)

Após a linearização através de regressão linear, de acordo com a equação de Arrhenius (Equação 4), foi calculada a energia de ativação aparente $\left(E_{\mathrm{a}}\right)$.

$$
\ln W_{c o r r}=\frac{-E a}{R T}+\ln \mathrm{A}
$$

em que $W_{\text {corr }}$ é a taxa de corrosão, $E_{\mathrm{a}}$ é a energia de ativação aparente, $A$ é o fator de frequência, $T$ é a temperatura absoluta e $R$ é a constante molar dos gases.

Na regressão linear foram obtidos bons valores de coeficiente de correlação, que são 0,9958 e 0,9986, para o experimento na ausência e na presença de extrato, respectivamente.
De forma complementar a equação de Arrhenius, a entalpia de ativação aparente $\left(\Delta H^{*}\right)$ e a entropia de ativação aparente $\left(\Delta S^{*}\right)$ podem ser calculadas a partir da equação de Eyring, que é mostrada na Equação 5:

$$
\ln \frac{W_{\text {corr }}}{T}=\ln \left[\frac{K_{B}}{h} e^{\Delta S^{* / R}}\right]-\frac{\Delta H^{*}}{R T}
$$

em que $W_{\text {corr }}$ é a taxa de corrosão, $K_{\mathrm{B}}$ é constante de Boltzmann, $h$ é a constante de Planck, $\Delta H^{*}$ é a entalpia de ativação e $\Delta S^{*}$ é a entropia de ativação.

A $E_{\mathrm{a}}, \Delta H^{*}$ e $\Delta S^{*}$ obtidas para o processo de corrosão, na solução ácida, na ausência e na presença de extrato podem ser observadas na Tabela 3.

Tabela 3. Energia de ativação, entalpia e entropia aparentes associados aos processos de corrosão em meio de $\mathrm{H}_{2} \mathrm{SO}_{4} 1 \mathrm{~mol} \mathrm{~L}^{-1}$, em presença e ausência do extrato hidro alcoólico de $M$. arboreus na concentração de 500 ppm

\begin{tabular}{lccc}
\hline & $E_{\mathrm{a}}\left(\mathrm{kJ} \mathrm{mol}^{-1}\right)$ & $\Delta H^{*}\left(\mathrm{~kJ} \mathrm{~mol}^{-1}\right)$ & $\Delta S^{*}\left(\mathrm{~J} \mathrm{~K}^{-1} \mathrm{~mol}^{-1}\right)$ \\
\hline Branco & 37,81 & 35,08 & $-236,71$ \\
$500 \mathrm{ppm}$ & 64,61 & 61,85 & $-180,59$ \\
\hline
\end{tabular}

Na Tabela 3 é possível observar o aumento nos valores de $E_{\mathrm{a}} \mathrm{e}$ $\Delta H^{*}$ na presença de extrato, isto é, a energia de barreira necessária para iniciar um processo corrosivo é maior, o que justifica a ação inibidora do extrato. Após aumento da temperatura, foi observada uma diminuição na eficiência de inibição da corrosão (Tabela 2), o que pode ser devido a diminuição das interações eletrostáticas entre o inibidor e a superfície. ${ }^{38-43}$

Foram observados valores negativos de $\Delta S^{*}$ tanto na ausência quanto na presença de extrato, porém, um valor menos negativo na presença do extrato. ${ }^{36,44}$

\section{Análise de microscopia eletrônica de varredura}

A Figura 6 apresenta os resultados das análises de microscopia eletrônica de varredura para superfície metálica, após 24 horas de imersão em solução de $\mathrm{H}_{2} \mathrm{SO}_{4} 1 \mathrm{~mol} \mathrm{~L}^{-1}$, na presença de $500 \mathrm{ppm}$ de extrato (Figura 6A), na ausência de extrato (Figura 6B) e antes da imersão (Figura 6C). Na Figura 6B é possível observar a superfície rugosa e irregular, caracterizando a deterioração do metal, proveniente do processo corrosivo e formação dos produtos de corrosão. Na presença de extrato (Figura 6A), a superfície é homogênea e com menor rugosidade, confirmando o caráter inibidor do extrato, observado nos ensaios gravimétricos. ${ }^{21-23}$

\section{CONCLUSÃO}

O extrato de $M$. arboreus apresentou boa eficiência de inibição da corrosão frente ao aço-carbono AISI 1020 em meio de ácido sulfúrico 1 mol L ${ }^{-1}$. Nos estudos de eficiência em função do tempo de imersão o extrato apresentou 97,5\% de eficiência de inibição da corrosão, nos tempos de $3 \mathrm{~h}, 24 \mathrm{~h}$ e $48 \mathrm{~h}$, na temperatura de $30^{\circ} \mathrm{C}$ e concentração de 500 ppm. Nessa mesma concentração, bons valores de eficiência também foram observados nas temperaturas de $40^{\circ} \mathrm{C}, 50^{\circ} \mathrm{C}$ e $60^{\circ} \mathrm{C}$, $96,5 \%, 96 \%$ e $93,5 \%$, respectivamente.

Os valores dos parâmetros termodinâmicos de $E_{\mathrm{a}} \mathrm{e} \Delta H^{*}$ corroboraram com a eficiência de inibição da corrosão, pois na presença de extrato ambos os valores foram maiores que em sua ausência, isto é, a energia de barreira necessária para iniciar um processo corrosivo é maior, o que justifica a ação inibidora do extrato. 


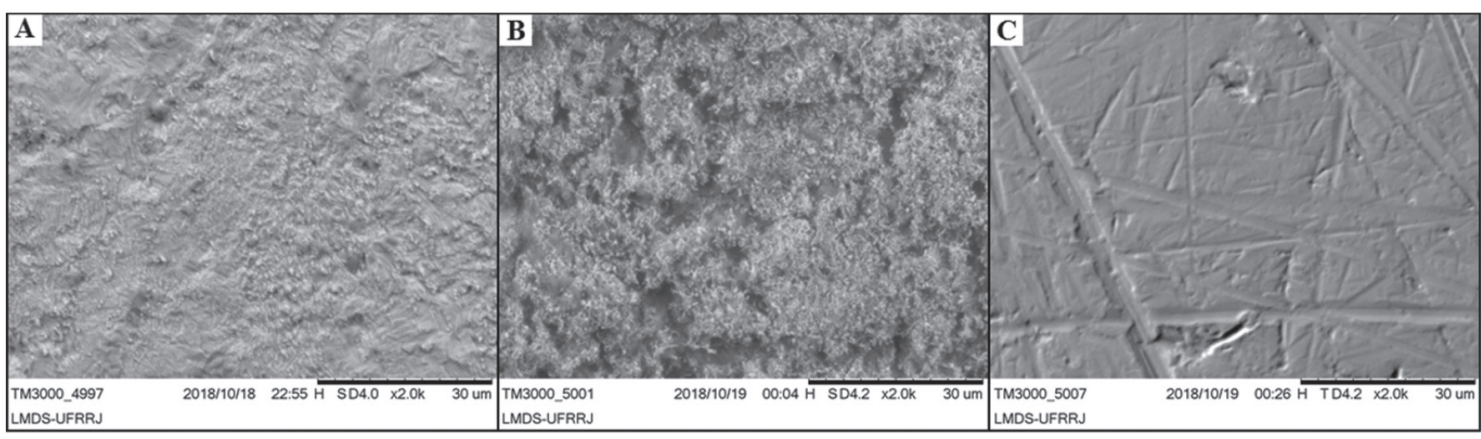

Figura 6. Imagem de microscopia eletrônica de varredura $(2000 \times)$ do aço carbono AISI 1020 imerso em $\mathrm{H}_{2} \mathrm{SO}_{4} 1 \mathrm{~mol} \mathrm{~L}^{-1}$ na presença de 500 ppm de extrato de $M$. arboreus. (A), ausência de extrato (B) e superfície antes da imersão $(C)$

De acordo com a prospecção realizada utilizando as técnicas espectroscópicas de infravermelho, $\mathrm{RMN}$ de ${ }^{1} \mathrm{He}^{13} \mathrm{C}$, concluiu-se que a eficiência anticorrosiva observada foi compatível com a presença de metabólitos da classe das porfirinas pirrolícas, o que justifica a capacidade quelante do extrato de $M$. arboreus.

Dessa forma, pode-se dizer que o extrato de $M$. arboreus é um potencial inibidor de corrosão verde, eficiente e estável tanto na variação de tempo, quanto de temperatura.

\section{AGRADECIMENTOS}

O presente trabalho foi realizado com apoio da Coordenação de Aperfeiçoamento de Pessoal de Nível Superior - Brasil (CAPES) Código de Financiamento 001. Os autores agradecem também ao Conselho Nacional de Desenvolvimento Científico e Tecnológico (CNPq), Fundação de Amparo à Pesquisa do Estado do Rio de Janeiro (FAPERJ), Petróleo Brasileiro S.A. (Petrobras) e ao projeto P\&D ANEEL-SUPERCABO.

\section{REFERÊNCIAS}

1. Gentil, V.; Corrosão, $5^{\text {a }}$ ed., LTC: Rio de Janeiro, 2007.

2. Rossi, C. G. F. T.; Scatena Júnior, H.; Maciel, M. A. M.; Dantas, T. N. C.; Quim. Nova 2007, 30, 1128.

3. Verma, C.; Olasunkanmi, L.; Ebenso, E. E.; Quraishi, M.; J. Mol. Liq. 2017, 251, 100 .

4. Li, X.; Xie, X.; Deng, S.; Du, G.; Corros. Sci. 2014, 87, 27.

5. Garai, S.; Garai, S.; Jaisankar, P.; Singh, J. K.; Elango, A.; Corros. Sci. 2012, 60, 193.

6. Umorem, S.A.; Eduok, U. M.; Carbohydr. Polym. 2016, 140, 314.

7. Hu, K.; Zhuang, J.; Zheng, C.; Ma, Z.; Gu, H.; Zeng, X.; Ding, J.; J. Mol. Liq. 2016, 222, 109.

8. Costa, D. C.; Costa, H.; Albuquerque, T. G.; Ramos, F.; Castilho, M. C.; Sanches-Silva, A.; Trends Food Sci. Technol. 2015, 45, 336.

9. Mari, M.; Bautista-Baños, S.; Sivakumat, D.; Postharvest Biol. Technol. 2016, 122,70 .

10. Torres, V. V.; Amado, R. S.; Sá, C. F.; Fernandez, T. L.; Riehl, C. A. S.; Torres, A. G.; D’Elia, E.; Corros. Sci. 2011, 53, 2385.

11. Ostovari, A.; Hoseinieh, S. M.; Peikari, M.; Shadizadeh, S. R.; Hashemi, S. J.; Corros. Sci. 2009, 51, 1935.

12. Abiola, O. K.; Otaigbe, J.; Corros. Sci. 2009, 51, 2790.

13. Singh, A.; Quraishi, M. A.; Arabian J. Chem. 2016, 9, S1584.

14. Deng, D.; Li, X.; Corros. Sci. 2012, 64, 253.

15. Krishnaveni, K.; Ravichandran, J.; Trans. Nonferrous Met. Soc. China 2014, 24, 2704

16. Singh, M. R.; Singh, G.; J. Mater. Environ. Sci. 2012, 3, 698.

17. Abel-Rahman, H. H.; Hafez, A. M.; Helmy, A. A.; Electrochemistry 2015, 83,440 .
18. Ammer, M. A. M.; Ferky, A. M.; Turk. J. Chem. 2015, 39, 1078.

19. Molina-Ocampo, L. B.; Valladares-Cisneros, M. G.; GonzalezRodrigues, J. G.; Int. J. Electrochem. Sci. 2015, 10, 388.

20. Carlos, M. L. F. P.; Valbon, A.; Neves, M. A.; Santos, M. R. L.; Echevarria, A.; J. Braz. Chem. Soc. 2018, 29, 2542.

21. Valbon, A.; Neves, M. A.; Echevarria, A.; Int. J. Electrochem. Sci. 2017, 12,3072

22. Valbon, A.; Neves, M. A.; Echevarria.; Mater. Res. 2018, 21, e20170847.

23. Silva, T. M. S.; Camara, C. A.; Barbosa-Filho, J. M.; Giulietti, A. M.; Quim. Nova 2010, 33, 571 .

24. Amorim, A. P. O.; Ribeiro, C., Almir, J.; Lopes, N. P., Castro, R. N.; Oliveira, M. C. C. O.; Carvalho, M. G.; Food Chem. 2014, 160, 204.

25. de Albuquerque, M. A.; Goulart, C. M.; Amorim, A. P. O.; de Oliveira, M. C. C.; Echevarria, A.; Rev. Virtual Quim. 2013, 5, 734.

26. Amorim, A. P. O.; de Oliveira, M. C. C.; Amorim, T. A.; Echevarria, A.; Antioxidants 2013, 2, 90.

27. Gupta, N. K.; Verma, C.; Quhaishi, M. A.; Mukherjee, A. K.; J. Mol. Liq. 2016, $215,47$.

28. Soliman, S. A.; Metwally, M. S.; Selim, S. R.; Bedair, M. A.; Abbas, M. A.; J. Ind. Eng. Chem. 2014, 20, 4311.

29. Hamani, H.; Douadi, T.; Daoud, D.; Al-Noaimi, M.; Rikkouh, R. A.; Chafaa, S.; J. Electroanal. Chem. 2017, 801, 425.

30. Torres, V. V.; Cabral, G. B.; Silva, A. C. G.; Ferreira, K. C. R.; D’Elia, E.; Quim. Nova 2016, 39, 423.

31. Li, X. H.; Deng, S. D.; Fu, H.; J. Appl. Electrochem. 2010, 40, 1641.

32. Ferreira, K. C. R.; Cordeiro, R. F. B.; Nunes, J. C.; Orofino, H.; Magalhães, M.; Torres, A. G.; D'Elia, E.; Int. J. Electrochem. Sci. 2016, 11, 406.

33. Rocha, J. C.; Gomes, J. A. C. P.; D'Elia, E.; Mater. Res. 2014, 17, 1581.

34. Souza, E. C. C. A.; Ripper, B. A.; Perrone, D.; D’Elia, E.; Mater. Res.2016, 19, 1276.

35. Trindade, R. S.; Santos, M. R.; Cordeiro, R. F. B.; D’Elia, E.; Green Chem. Lett. Rev. 2017, 10, 444.

36. Rocha, J. C.; Gomes, J. A. C. P.; D’Elia, E.; Gil Cruz, A. P.; Cabral, L. M. C.; Torres, A. G.; Monteiro, M. V. C.; Int. J. Electrochem. Sci. 2012 , 7, 11941.

37. Barros, I. B.; Kappel, M. A. A.; Santos, P. M.; Junior, V. F. V.; D'Elia, E.; Bastos, I. N.; Mater. Res. 2016, 19, 187.

38. Rocha, J. C.; Gomes, J. A. C. P.; D’Elia, E.; Corros. Sci. 2010, 52, 2341.

39. Teixeira, V. M.; dos Santos, E. C.; Rezende, M. J. C.; D’Elia, E.; Rev. Virtual Quim. 2015, 7, 1780.

40. Oguzie, E. E.; Corros. Sci. 2008, 50. 2998.

41. Satapathy, A. K.; Gunasekaran G.; Sahoo, S. C.; Amit, K.; Rodrigues, P. V.; Corros. Sci. 2009, 51, 2848.

42. El-Etre, A. Y.; Mater. Chem. Phys. 2008, 108, 278.

43. Nathiya, R. S.; Raj, V.; Egypt. J. Pet. 2017, 26, 313.

44. Badra, E. A.; Bedairb, M. A.; Shabana, S. M.; Mater. Chem. Phys. 2018, 219,444 